\title{
Um StoryBot como apoio a processos de autoaprendizagem através de desafios lógicos matemático-computacionais e valorização cultural
}

\author{
Larissa Pessoa $^{1}$, Lia Martins ${ }^{1}$, Gustavo Martins ${ }^{1}$, Victória Guimarães ${ }^{1}$, \\ Rosiane de Freitas ${ }^{1}$ \\ ${ }^{1}$ Instituto de Computação - Universidade Federal do Amazonas (UFAM) \\ Manaus - AM - Brazil \\ \{lsp, lia.martins, gfm, vsg, rosiane\}@icomp.ufam.edu.br
}

\begin{abstract}
The concern to provide more effective ways of learning that involve and motivate young students has moved academia and the education industry. Going where young people already like to be and using the same rules of the game are premises of this proposal. Thus, through the Design Thinking approach, a Python 3.0 chatbot was developed on the Discord platform based on narratives from Brazilian folklore, a StoryBot, structured in phases based on the pillars of computational thinking and with mathematical-computational logical challenges that promote the manipulation of CS concepts. An action-research with students from the end of elementary school was conducted showing the tools' potential in self-learning processes mediated by educators.
\end{abstract}

Resumo. Prover formas efetivas de aprendizagem que envolvam e motivem os jovens estudantes tem movimentado a academia e a indústria da educação. Através de uma abordagem baseada no Design Thinking foi desenvolvido um chatbot em Python para a plataforma Discord baseando-se em narrativas do folclore brasileiro, estruturado em capítulos, cada um baseado nos pilares do pensamento computacional e com desafios lógicos matemático-computacionais que promovem a manipulação de conceitos de ciência da computação. Uma pesquisa-ação com alunos do final do ensino fundamental foi conduzida mostrando o potencial de tal estratégia em processos de autoaprendizagem mediado por educadores.

\section{Introdução}

Uma estratégia de aprendizagem para atrair a atenção dos alunos, tem sido a contação de histórias e seus enredos e personagens envolventes, pois estimula a criatividade e a imaginação, facilita o aprendizado, trabalha o senso crítico e valores morais, e ainda pode explorar a cultura e a diversidade [de Sousa and Dalla Bernardino 2011]. Com o aumento do acesso à Internet via smartphones e o uso de aplicativos de mensagens, ferramentas de conversação para tomada de decisão, os chatbots, têm tido um crescimento extraordinário [Bahja et al. 2020] [Sandu and Gide 2019].

Deste modo, uma questão motivou a presente investigação: será que um jogo baseado em contação de histórias e desafios lógicos, usando uma plataforma já dominante entre os jovens, pode ter um forte potencial de apoio à aprendizagem a ser explorado? 
Sendo assim, neste trabalho está sendo proposta a criação de um jogo baseado em narrativa interativa programada com múltiplos caminhos de aprendizagem, onde trabalham-se os quatro pilares do pensamento computacional através de desafios lógicos matemáticocomputacionais, viabilizado através de um chatbot para a plataforma Discord.

O artigo está organizado como segue. Na Seção 2 é dada a fundamentação teórica sobre narrativas em jogos, chatbot e pensamento computacional. Na Seção 3, são apresentados alguns trabalhos relacionados a adoção de chatbots e contação de histórias em processos de aprendizagem. Na Seção 4 são descritos a metodologia de pesquisa e o processo de concepção e desenvolvimento do jogo. Durante a seção 5 são apresentados o enredo, a definição dos conteúdos que foram trabalhos em cada desafio e o detalhamento do processo de prototipação. Na Seção 6, a pesquisa-ação conduzida com estudantes do ensino fundamental é descrita e analisada. Por fim, na Seção 7 são tecidas considerações finais sobre a proposta e alguns trabalhos futuros que poderão ser desenvolvidos a partir do trabalho atual.

\section{Fundamentação Teórica}

Nesta seção serão apresentados os conceitos que fundamentam esta pesquisa, envolvendo storytelling, aprendizagem (não-) Linear e pensamento computacional.

Narrativa ou Storytelling nada mais é do que a arte de contar histórias. Antes mesmo do desenvolvimento da escrita, as pessoas contavam histórias umas às outras. Não apenas como forma de entretenimento mas também como ferramenta de aprendizagem. A narrativa permite ao indivíduo obter um sentido para a experiência. Dessa forma, o uso dessa ferramenta estimula a criatividade e facilita a compreensão de assuntos complexos, sobretudo para os mais jovens. Além disso, o contato com histórias contribui para o desenvolvimento das habilidades de comunicação, organização de ideias, de pensamento crítico, e também fornece ferramentas para que novas histórias sejam criadas [Robin 2016]. Narrativas não-lineares exploram múltiplos caminhos com base em tomadas de decisão, promovendo interatividade e uma participação ativa do leitor no processo [Liu et al. 2010].

Uma das maneiras de se contar esse tipo de história é através de um chatbot. Se trata de uma ferramenta de software que interage com os usuários em um determinado tópico ou em um domínio específico de uma forma natural e coloquial, usando texto e voz [Smutny and Schreiberova 2020]. De forma geral, Chatbots têm por objetivo, por exemplo, responder às perguntas de tal forma que se tenha a impressão de estar conversando com outra pessoa, e não com um programa de computador [Antunes et al. 2018]. Em educação, tem sido utilizados nas mais variadas áreas de ensino sobretudo na forma do uso de bots que simulam um diálogo humano no processo de ensino/aprendizagem [Bulhoes et al. 2020].

Além disso, é crescente a preocupação com a criação de novas maneiras de se aprender e ensinar, uma vez que estimular habilidades para resolução de problemas é essencial para o cidadão do século XXI, assim nasce o conceito de Pensamento Computacional (do inglês, computacional thinking - CT). Diferentes fontes definem o CT como uma lista de conceitos e competências, que apoiam e diferem entre si. As habilidades mais comuns dizem respeito à abstração, algoritmos, reconhecimento de padrão, decomposição de problemas, paralelismo, depuração, teste e estrutura de con- 
trole [Zhang and Nouri 2019].

\section{Trabalhos relacionados}

Alguns trabalhos surgiram em decorrência da criação de Ferramentas de Autoria (FAS), utilizando diferentes plataformas para exibição de narrativas não-lineares e ferramentas educacionais computacionais. Na literatura, encontram-se trabalhos onde são apresentadas histórias interativas não lineares e ferramentas de aprendizagem que podem ser acessadas em diversas ferramentas, e que exploram no seu desenvolvimento os quatro pilares do pensamento computacional. Também encontram-se produções que utilizam chatbot para interagir com o usuário realizando uma determinada função. Na Tabela 1 pode-se verificar os trabalhos relacionados e quais características foram avaliadas.

Tabela 1. Quadro comparativo dos trabalhos relacionados da literatura. Fonte: própria.

\begin{tabular}{|c|c|c|c|c|c|}
\hline Título & $\begin{array}{l}\text { Ferramenta/ } \\
\text { Plataforma }\end{array}$ & Aplicação & PC & $\begin{array}{l}\text { Narrativa } \\
\text { não linear }\end{array}$ & Chatbot \\
\hline $\begin{array}{l}\text { CT Puzzle Test: Em direção a uma avaliação } \\
\text { interativa do pensamento computacional. } \\
\text { [Raabe et al. 2020] }\end{array}$ & $\begin{array}{l}\text { Online, } \\
\text { implementado } \\
\text { com HTML5 e } \\
\text { Javascript }\end{array}$ & Site & SIM & NÃO & NÃO \\
\hline $\begin{array}{l}\text { Nonlinear Storytelling Approach to Developing } \\
\text { Computational Thinking Skills. } \\
\text { [Letonsaari et al. 2021] }\end{array}$ & Twine & Serious Game & SIM & SIM & NÃO \\
\hline $\begin{array}{l}\text { Chatbots for learning: A review of educational } \\
\text { chatbots fo the Facebook Messenger. } \\
\text { [Smutny and Schreiberova 2020] }\end{array}$ & $\begin{array}{l}\text { Facebook } \\
\text { Messenger }\end{array}$ & - & NÃO & NÃO & SIM \\
\hline $\begin{array}{l}\text { Digital storytelling with chatbots: } \\
\text { mapping L2 participation and perception patterns. } \\
\text { [Bailey et al. 2021] }\end{array}$ & $\begin{array}{l}\text { Facebook } \\
\text { Messenger }\end{array}$ & - & NÃO & SIM & SIM \\
\hline $\begin{array}{l}\text { Learning During COVID-19 Pandemic: } \\
\text { Online Education Community, Based on Discord. } \\
\text { [Vladoiu and Constantinescu 2020] }\end{array}$ & Discord & $\begin{array}{l}\text { Sala de aula } \\
\text { online }\end{array}$ & NÃO & NÃO & NÃO \\
\hline $\begin{array}{l}\text { Zoe em uma viagem ao mundo computacional } \\
\text { perdido na Amazônia }\end{array}$ & $\begin{array}{l}\text { Discord, } \\
\text { implementado } \\
\text { com Python }\end{array}$ & Chatbot ZoAm & SIM & SIM & SIM \\
\hline
\end{tabular}

Como na Tabela 1, de acordo com o trabalho de Raabe [2020] é proposta a criação e aplicação de testes de PC baseados em puzzles, onde busca estimular o raciocínio lógico e o pensamento crítico. Em Letonsaari [2021] é feito a investigação de narrativas não lineares como meio de ensino de habilidades fundamentais do PC. Já no trabalho de Smutny [2020] e em Bailey [2021], foram examinados chatbots educacionais que apoiam aprendizagem em sala de aula. Por fim, o trabalho de Vladoiu [2020], utilizou o Discord para manter contato com os alunos de graduação devido a pandemia causada por Covid-19, utilizando como ferramenta de ensino. A história interativa não linear "Zoe em uma viagem ao mundo computacional perdido na Amazônia" é proposta no presente trabalho, onde os caminhos escolhidos pelo usuário levam a desafios que exploram os pilares do pensamento computacional, bem como conceitos computacionais mais complexos. A história é apresentada na ferramenta Discord com uso de chatbot, visto que é uma plataforma para troca de mensagens de texto, áudio e vídeo instantânea e facilita a criação de chatbots para interação com os usuários. 


\section{Metodologia de Desenvolvimento do StoryBot Discord ZoeAm}

Nesta seção é apresentada a metodologia utilizada para todo o desenvolvimento do projeto, posteriormente a metodologia seguida para o desenvolvimento da história interativa não linear programada do jogo intitulado: "ZoeAm: uma viagem a um mundo computacional perdido na Amazônia" e por fim, a técnica adotada para análise dos dados do experimento com o jogo.

A produção do jogo foi baseada nas técnicas de design thinking, envolvendo três etapas principais: (1) imersão - entendimento do problema e suas implicações; (2) ideação - abordagem para se atacar o problema; e, (3) prototipagem - passagem dos conceitos abstratos para o sistema ou produto desejado, de forma a representar a realidade - mesmo que simplificada e proporcionar validações. Durante a fase de ideação foi concebida a ideia de uma narrativa estruturada em quatro capítulos, cada um trabalhando um pilar do CT somado a outros conteúdos computacionais ou pedagógicos para exemplificar o pilar correspondente. Além disso, preocupou-se em ter uma personagem central feminina que teria o CT altamente desenvolvido, suas habilidades seriam mostradas nas soluções de problemas que surgissem no decorrer da história. A temática regional foi escolhida para valorizar toda a diversidade e beleza da região da Amazônia.

Os elementos da narrativa foram inseridos no enredo seguindo a estrutura da Jornada do Herói. O autor em [Campbell 2008] define esse padrão na seguinte forma: "Um herói se aventura além do mundo comum, para uma região de maravilhas sobrenaturais: forças fabulosas são encontradas e uma vitória decisiva é conquistada: o herói volta desta aventura misteriosa com o poder de espalhar um novo conhecimento a seus semelhantes." (tradução nossa). Ou seja, o modelo envolve um herói que vai numa aventura, aprende uma lição, ganha uma vitória com esse novo conhecimento, e depois volta para casa transformado. A jornada do herói consiste em três etapas principais. A primeira, referida como A Partida, trata-se do início da história, o herói está vivendo no chamado "mundo comum"quando recebe um chamado à aventura. Na segunda etapa, A Iniciação, o herói entra no mundo fantástico, onde ele deve enfrentar uma série de tarefas até atingir o clímax da história - o principal obstáculo ou inimigo. Então, o herói deve colocar em prática tudo o que aprendeu durante sua jornada para superar o obstáculo. E por fim, na terceira etapa, O Retorno, sentindo que está pronto para voltar ao seu mundo, o herói deve partir. Uma vez de volta ao mundo comum, ele passou por uma transformação pessoal na realização de sua aventura e isso o mudou como pessoa.

A metodologia utilizada para a validação do chatbot foi baseada em dois modelos de avaliação de jogos: 1) Desurvire e Wiberg e 2) korhonen e Koivisto. Os dois modelos são detalhados na tabela 2:

Tabela 2. Definições dos modelos de avaliação de jogos que foram base para o método de avaliação usado para o StoryBot ZoAM.

\begin{tabular}{|lll|}
\hline \multicolumn{1}{|c|}{ Modelo } & \multicolumn{1}{c}{ Definição } & \multicolumn{1}{c}{$\begin{array}{c}\text { Divisão por categoria } \\
\text { das Heurísticas }\end{array}$} \\
\hline $\begin{array}{l}\text { Desurvire } \\
\text { e Wiberg }\end{array}$ & $\begin{array}{l}\text { Estabeleceram um conjunto de heurísticas que foram adaptadas de software de produtividade para jogos, } \\
\text { gerando uma lista refinada, Heurística de Jogabilidade (PLAY), que pode ser aplicada mais cedo no } \\
\text { desenvolvimento do jogo, bem como ajudar os desenvolvedores entre pesquisas formais de usabilidade/ } \\
\text { jogabilidade durante o ciclo de desenvolvimento [Desurvire and Wiberg 2009]. }\end{array}$ & $\begin{array}{l}\text { I) Jíne Frieza/Entretenimento/ } \\
\text { Humor/Imersão emocional; } \\
\text { III) Usabilidade e mecânica. }\end{array}$ \\
\hline \multirow{2}{*}{$\begin{array}{l}\text { korhonen } \\
\text { e Koivisto }\end{array}$} & $\begin{array}{l}\text { Definiram um modelo de avaliação com heurísticas voltadas para dispositivos móveis, onde o maior ganho } \\
\text { aqui são as heurísticas de mobilidade, que são definidas pela facilidade com que o jogo permite que um }\end{array}$ & I) Jogabilidade; \\
& $\begin{array}{l}\text { jogador entre no mundo do jogo e como ele se comporta em ambientes diversos e inesperados } \\
\text { [Korhonen and Koivisto 2006]. }\end{array}$ & II) Usabilidade; \\
III) Mobilidade.
\end{tabular}


Tabela 3. Heurísticas de usabilidade, jogabilidade/imersão e mobilidade aplicadas no gamebot ZoAm.

\begin{tabular}{|c|c|}
\hline Categoria & Heurística \\
\hline \multirow{3}{*}{ I - Usabilidade } & H1. Os jogadores devem receber ajuda contextual enquanto jogam para que eles não fiquem presos e precisam confiar em um manual para obter ajuda. \\
\hline & H2. O jogador recebe controles básicos o suficiente para aprender rapidamente, mas expansível para opções avançadas para jogadores avançados. \\
\hline & H3. O layout da tela é eficiente, integrado e visualmente agradável. \\
\hline \multirow{7}{*}{$\begin{array}{l}\text { II - Jogabilidade } \\
\text { e Imersão }\end{array}$} & H4. Os jogadores acham o jogo divertido, sem tarefas repetitivas ou entediantes. \\
\hline & H5. Os jogadores não devem ser penalizados repetidamente pela mesma falha. \\
\hline & H6. O ritmo do jogo é aplicado para aplicar pressão \\
\hline & sem frustrar os jogadores. O nível de dificuldade varia para que os jogadores experimentem desafios melhores à medida que desenvolvem maestria. \\
\hline & H7. Os desafios são experiências de jogo positivas, em vez de experiências , resultando em querer jogar mais, em vez de desistir. \\
\hline & H8. O mundo do jogo reage ao jogador e se lembra de sua passagem através dele. \\
\hline & H9. Os objetivos do jogo são claros. O jogo oferece objetivos claros, apresenta superando metas no início, bem como metas de curto prazo durante o jogo \\
\hline \multirow{3}{*}{ III - Mobilidade } & H10. Ao ligar o jogo, o jogador tem informações suficientes para começar a jogar rapidamente. \\
\hline & H11. A navegação é consistente, lógica e minimalista. \\
\hline & jogo não apresenta falhas ou inconsistências. \\
\hline
\end{tabular}

Os dois modelos envolvem uma série de heurísticas, entretanto, para o StoryBot ZoAM reduzimos o número de heurísticas utilizadas e mesclamos os dois modelos, uma vez que por se tratar de chatbot algumas não eram aplicáveis. As heurísticas escolhidas podem ser vistas na tabela 3 .

\section{ZoeAm: Uma viagem a um mundo computacional perdido na Amazônia}

Nesta seção são apresentados o enredo, a definição dos conteúdos que foram trabalhos em cada desafio bem como detalhamos o processo de prototipação do jogo.

O Enredo da história se passa na região amazônica. Zoe, a personagem principal, é uma jovem engenheira que decidiu fazer um passeio de barco pelo rio Amazonas. Zoe estava no terceiro andar da embarcação quando ficou admirada com o Encontro das Águas e um boto cor de rosa, assim, acabou caindo da embarcação e se afogando nos rios. Quando ela acordou já estava na beira de um rio, chovia muito forte e por isso procurou uma caverna para se abrigar até que se deparou com caminhos dentro da caverna, um deles, levando a cidade escondida no meio da Floresta Amazônica mas altamente desenvolvida tecnologicamente. Assim, os nativos constroem uma aliança com Zoe para que ela os ajude a resolver a situação da barreira de proteção, dessa forma, todos sairiam ganhando e Zoe poderia voltar para casa. Durante a jornada, Zoe tem diversos encontros com seres do folclore brasileiro, mas que na cidade misteriosa são seres inteligentes com grandes conhecimentos sobre desenvolvimento sustentável, surgem como mentores, desafiando e testando Zoe a cada momento.

Dessa forma, temos que as principais etapas da jornada do herói foram representadas no enredo. A partida, quando Zoe passeia pelo rio Amazonas, configura o mundo comum onde a sua queda no Encontro das Águas trata da passagem do mundo comum para um mundo fantástico. Uma vez nesse mundo, Zoe recebe o chamado à aventura, por meio do pedido de ajuda dos habitantes desse outro mundo. A partir de então, Zoe deve cumprir uma série de tarefas sendo cada uma dessas dedicada a um aspecto do Pensamento computacional e também de preservação do meio ambiente, temos assim a segunda etapa da jornada. A história então atinge o clímax, onde Zoe deve solucionar um enigma através das lições aprendidas nas tarefas anteriores. Por fim, o retorno, Zoe tem de fazer uma escolha, optando por estimular aquele povo a contatar o mundo comum ou permanecerem isolados. Uma descrição mais detalhada pode ser observada na Figura 1. 


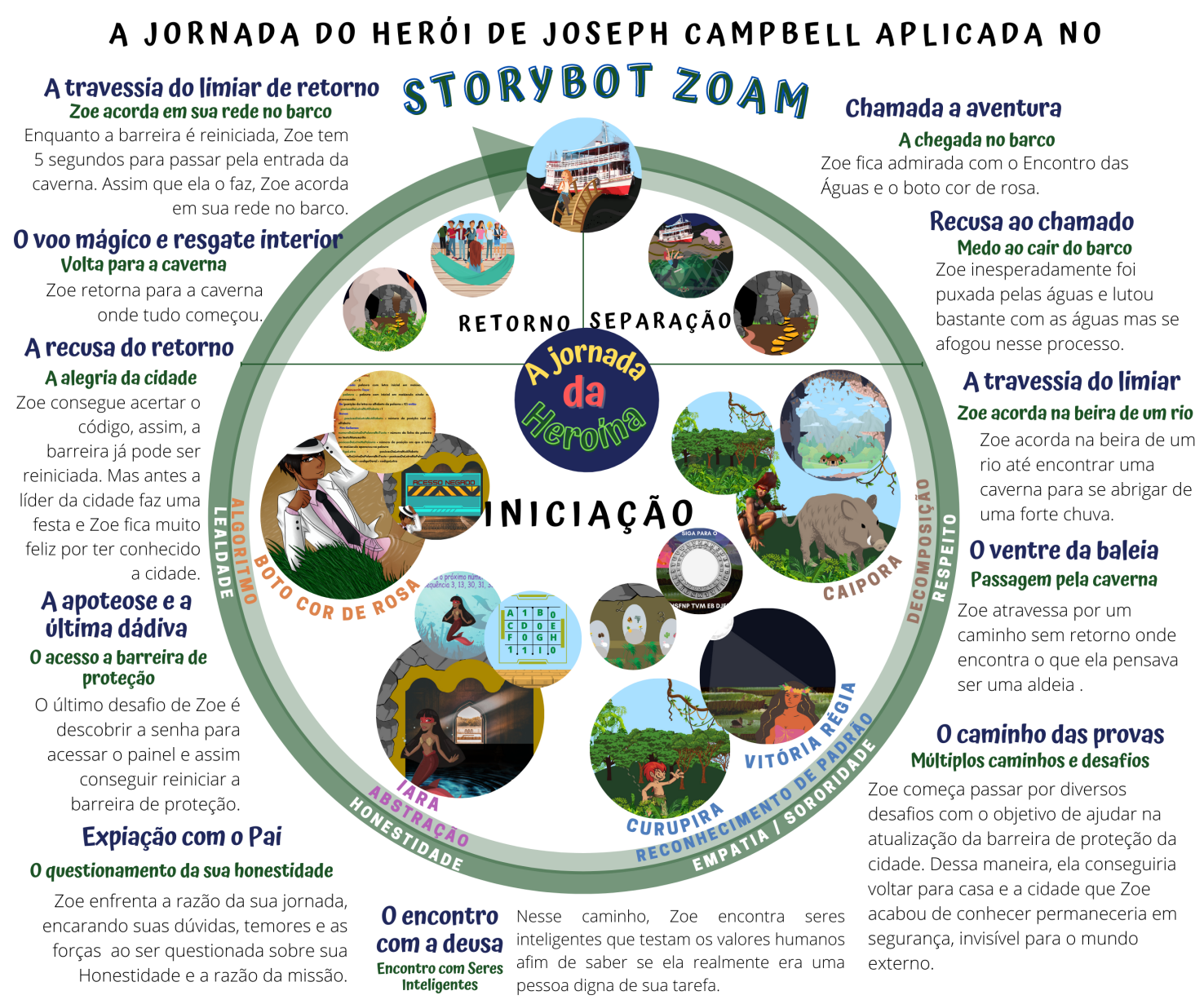

Figura 1. A jornada do Herói aplicada no StoryBot ZoAM.

$\mathrm{Na}$ Prototipação foram definidos detalhes de implementação da história e dos desafios e as decisões de projeto. Para a implementação foi escolhida a ferramenta Discord por esses motivos: a) A ferramenta possui interface web, de modo, que não é necessário fazer qualquer tipo de download para uma aplicação; b) é um ambiente muito comum para jovens da comunidade de jogos; c)Possui uma biblioteca chamada discord.py na linguagem de programação Python com ampla documentação; d)Possui um gerenciador de aplicações de fácil integração para o desenvolvimento de chatbots.

O chatbot foi desenvolvido através da linguagem de programação Python 3.0 e vinculado à plataforma de conversação multimídia Discord, sendo criado um servidor chamado ZoAm, por onde eventos ou mensagens são passados através do servidor para o chatbot por meio de uma conexão WebSocket permanente. Assim, o chatbot pode responder a esses eventos ou mensagens de cada usuário. Após a escrita da versão inicial da história e definição da plataforma, se deu o desenvolvimento seguindo as etapas conforme a figura 2 .

\section{Pesquisa-ação e Análise dos Resultados}

Os sujeitos desta pesquisa foram 20 estudantes, com idades entre 13 e 18 anos, sendo 10 meninos e 10 meninas, cursando o $8^{\circ}$ e $9^{\circ}$ ano do Ensino Fundamental, na Escola Municipal Jornalista Sabá Raposo, situada em um bairro periférico da cidade de Manaus/AM. 


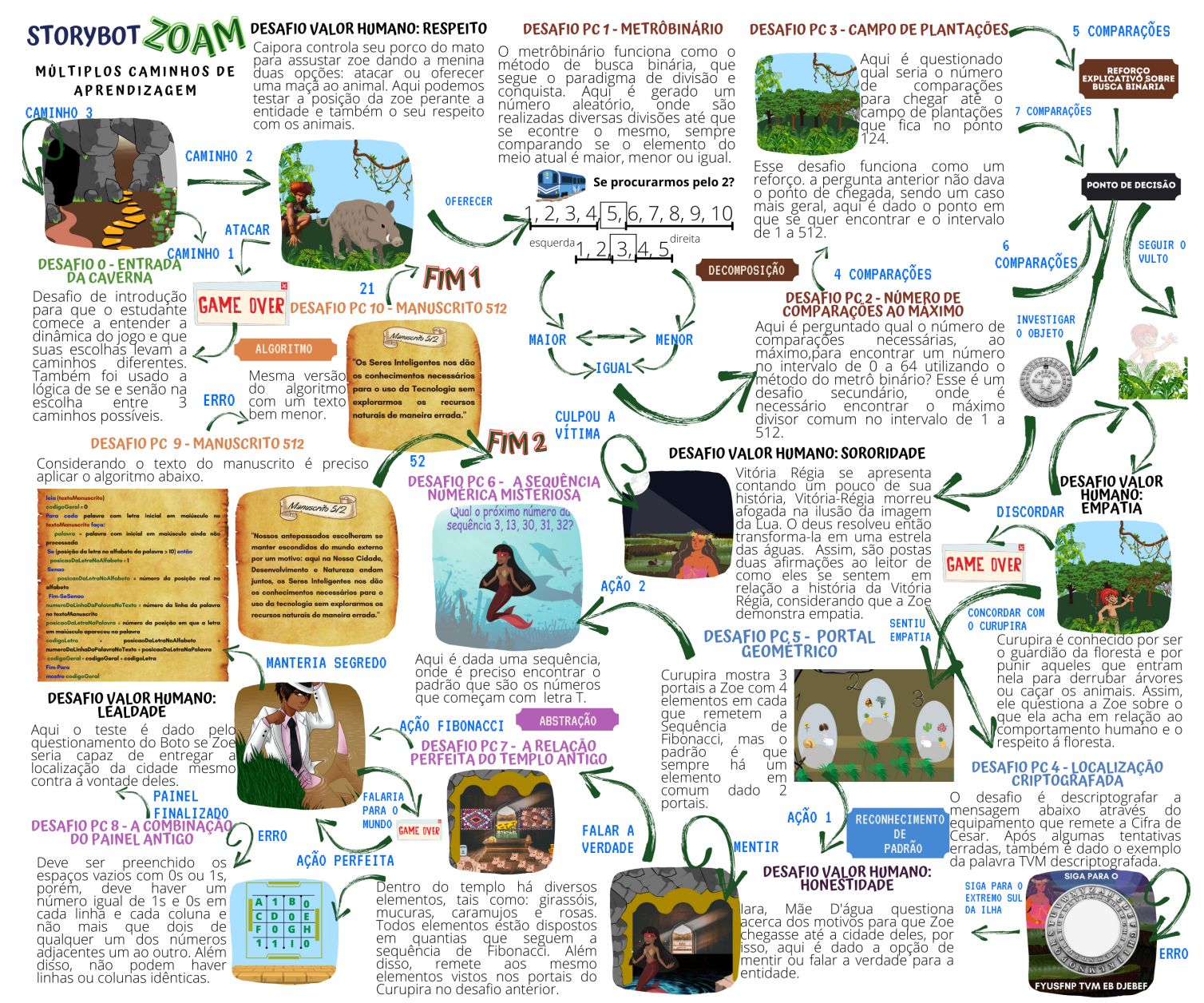

Figura 2. Múltiplos caminhos de aprendizagem do StoryBot ZoAm.

Tal escola foi escolhida por ser pública e possuir um laboratório com equipamentos e internet disponível para os aprendizes, requisito necessário para acessar o servidor ZoAm na ferramenta Discord.

Inicialmente, foi passado um vídeo tutorial para que os estudantes entendessem a dinâmica do StoryBot em relação aos comandos e interações. Após isso, foi indicado um número para cada estudante de 1 a 20 . Este número era equivalente ao seu canal no servidor ZoAm. Seguindo, os discentes digitaram um "oi" para o chatbot iniciar o jogo. Cada discente seguiu seu caminho no jogo de acordo com suas decisões, compreensão e aprendizagem que foram ocorrendo de acordo o que foi proposto pelo jogo. Os desafios e caminhos propostos podem ser observados na Figura 2. O experimento como um todo teve um período de $01: 30 \mathrm{~h}$, sendo que 20min foi para ligar o equipamento, logar na ferramenta Discord e acessar o servidor ZoAm, 01:00h para brincar no jogo e 00:10h para responder o formulário que foi disponibilizado no link: https://forms.gle/xnX3TL2PFTcphvLN8, para reportar como foi sua interação com o jogo. Dentre esses 20 estudantes, somente 4 finalizaram o jogo de forma efetiva, outros 6 chegaram bem perto e os restantes pararam em algum desafio anterior. Na Figura 3 é possível identificar os caminhos percorridos pelos estudantes, a média de tentativas e a média de tempo de resolução por desafio. Lembrando que para o desafio 1 não foi considerado o número de tentativas, uma vez que 
exigia a geração de um número aleatório que poderia tornar o caminho maior ou menor durante este desafio.

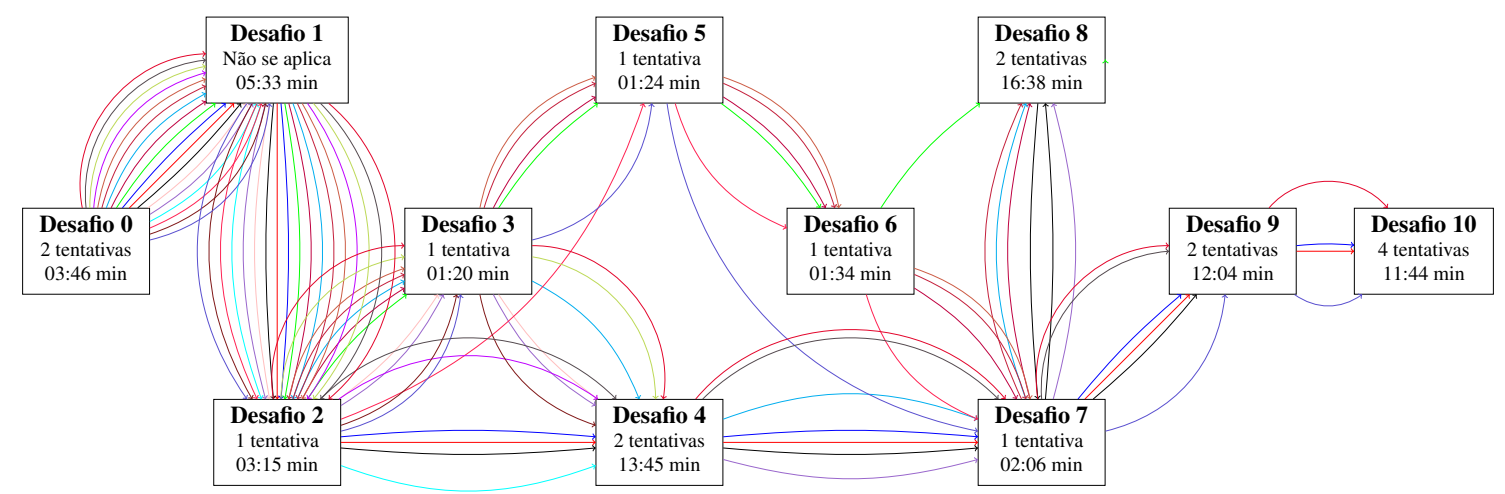

Figura 3. Multigrafo de representação dos caminhos não-lineares de aprendizagem, onde: cada vértice (retângulos) é um desafio do jogo e cada aresta (segmento de reta) é o caminho de um dos 20 alunos (cada cor é um aluno específico).

Pelos caminhos percorridos é possível perceber que os desafios 4 e 8 necessitam de um tempo médio de resolução bem superior aos demais, e que poderiam ser repensados para uma próxima experimentação prática, afim de não gerar gargalos permitindo que os estudantes consigam aprender e superem o desafio. Também foi observado que os estudantes tentavam discutir as soluções das questões entre si, porém, logo percebiam que tinham tomado caminhos diferentes com desafios distintos. Procuravam ajudar quando percebiam que já tinham passado por aquele caminho mas nem sempre a solução era a mesma.

Passando para discussão das questões respondidas no formulário com as perguntas adaptadas das heurísticas mostradas na tabela 3, iniciando a análise pelas contribuições das professoras que afirmaram nunca ter usado a ferramenta Discord em atividades escolares, mas a consideraram uma ferramenta intuitiva para utilizar em sala de aula. Apontaram alguns pontos, tais como: a conexão com a internet um tanto fraca para suportar os 20 equipamentos conectados e a dificuldade dos alunos em logar na ferramenta, como fatores que atrapalharam um pouco a aplicação do experimento. Porém, asseguraram que usariam o StoryBot ZoAM em sua sala de aula, pois a maioria dos educandos gostou de jogar. Identificaram no decorrer do jogo o contato dos alunos com operações matemáticas, raciocínio lógico e interpretação do texto.

Passando para a análise dos alunos, 50\% deles acessaram o jogo de um computador, $40 \%$ do tablet e $10 \%$ de celular próprio. Oito alunos indicaram ter grande familiaridade com o Discord e 5 participantes registraram não ter tido contato nenhum, os demais já tinham utilizado eventualmente. Verificando o tempo de uso de celular, vídeos e computador, $50 \%$ registraram passar entre 4 e 6 h por dia, 22,7\% menos de 2h, 13,6\% entre 6 e $10 \mathrm{~h}$, e $13,6 \%$ mais de $10 \mathrm{~h}$.

Na seção Usabilidade, a segunda do formulário, $87 \%$ dos alunos identificaram a existência do comando de ajuda e $78 \%$ registram que tiveram informações suficientes para iniciar o jogo, $87 \%$ concordaram que os comandos do chatbot estão condizentes com a opção realizada, $85 \%$ que os comandos estão claros para o leitor e $100 \%$ admitiram que 
as informações com o chatbot são visualmente agradáveis e integradas com o contexto da história. Olhando para o grau de dificuldade sentida na leitura dos textos, sendo 1 nenhuma e 5 muita dificuldade, 7 aprendizes marcaram a opção 1, 4 a opção 2, 6 a opção 3, 2 a opção 4 e 1 a opção 5, demonstrando que precisa-se adequar a linguagem do chatbot com vocabulário mais adequado para os jovens, pois houve alguma dificuldade com 13 alunos.

Na terceira Seção do formulário, a Jogabilidade, obteve-se que 56,5\% dos alunos afirmaram não achar os desafios e atividades realizadas durante a execução do chatbot entediante, $43,5 \%$ dos estudantes não se sentiram penalizados repentinamente por uma mesma falha e 73,9\% dos adolescentes sentiram que o nível de dificuldade dos desafios aumentou no decorrer do jogo. Continuando a observação da Seção de Jogabilidade, $54,5 \%$ dos discentes não acharam os desafios difíceis a ponto de serem frustrantes. Tal como, 60,9\% não sentiram que ficaram estagnados por um longo período de tempo. Por fim a esta Seção, 60,9\% dos aprendizes afirmaram que a narrativa foi transmitida de forma clara sem sentirem vontade de ignora-las.

Passando para a Seção Mobilidade do formulário, que lida com questões de interface uma vez que não pode-se controlar onde e quando os jogos são jogados. Introduz-se exibindo que 78,3\% dos estudantes alegaram que o chatbot pode ser iniciado rapidamente, $78,3 \%$ dos aulistas asseguraram que o chatbot funcionou bem no ambiente do Discord e por fim, 78,3\% dos aprendentes não encontraram algum problema técnico durante a execução do chatbot. Na última Seção do formulário, os estudantes tiveram a oportunidade de tecer comentários sobre o chatbot, tais como: "No geral, o jogo é muito bom. Adorei o roteiro com as lendas amazônicas e a forma como são inseridas. E as figuras também são bem legais.", outros fizeram comentários mais pontuais dando reais sugestões de como melhorar o jogo, como: "Muito bom, porém, deixar os comandos mais fáceis, por exemplo "!caipora cont"invés de "!caipora continuar", deixar os comandos mais resumidos é bem interessante :)".

\section{Considerações finais}

A pesquisa-ação efetuada para validar o sistema desenvolvido, mostrou o potencial da proposta uma vez que manteve motivado e imersivo um grupo de estudantes. A manipulação do jogo por estudantes do final do ensino fundamental propiciou a análise de diversas questões relacionados a facilidade de uso, assimilação dos conceitos embutidos nos desafios e, principalmente, o quão motivados e interessados os alunos ficaram sobre os conceitos e seus próprios desempenhos na atividade proposta.

Como continuação deste trabalho, também pretende-se expandir a história, incorporando outros aspectos regionais da amazônia e desafios mais elaborados, de forma a torná-la ainda mais envolvente e realizar uma nova aplicação abrangido um maior número de estudantes. Também será disponibilizado o StoryBot na loja de bots do Discord gratuitamente para que mais educadores e estudantes tenham acesso. Como trabalho futuro pretende-se realizar a publicação da mesma história no formato de livro físico interativo.

\section{Referências}

Antunes, E. E., Picchi, M. d. L. P., Pinto, G. S., and Ragazani, A. V. F. (2018). Chatbot como interface para a aprendizagem da língua inglesa. Revista Interface Tecnológica, 
15(1):28-38.

Bahja, M., Hammad, R., and Butt, G. (2020). A user-centric framework for educational chatbots design and development. In International Conference on Human-Computer Interaction, pages 32-43. Springer.

Bailey, D., Southam, A., and Costley, J. (2021). Digital storytelling with chatbots: Mapping 12 participation and perception patterns. Interactive Technology and Smart Education.

Bulhoes, D. B., Assis, L. P., Bodolay, A. N., Andrade, A. V., and Pitangui, C. G. (2020). Professora vitória: um chatbot para o ensino da leitura. In Anais do XXXI Simpósio Brasileiro de Informática na Educação, pages 451-460. SBC.

Campbell, J. (2008). The hero with a thousand faces, volume 17. New World Library.

de Sousa, L. O. and Dalla Bernardino, A. (2011). A contação de histórias como estratégia pedagógica na educação infantil e ensino fundamental. Educere et Educare, 6(12).

Desurvire, H. and Wiberg, C. (2009). Game usability heuristics (play) for evaluating and designing better games: The next iteration. In International conference on online communities and social computing, pages 557-566. Springer.

Korhonen, H. and Koivisto, E. (2006). Playability heuristics for mobile games. pages 9-16.

Letonsaari, M. et al. (2021). Nonlinear storytelling approach to developing computational thinking skills. Helsinki Studies in Education.

Liu, C.-C., Liu, K.-P., Chen, G.-D., and Liu, B.-J. (2010). Children's collaborative storytelling with linear and nonlinear approaches. Procedia-Social and Behavioral Sciences, 2(2):4787-4792.

Raabe, A., Viana, C., and Calbusch, L. (2020). Ct puzzle test: Em direção a uma avaliação interativa do pensamento computacional. In Anais do XXXI Simpósio Brasileiro de Informática na Educação, pages 1683-1692. SBC.

Robin, B. (2016). The power of digital storytelling to support teaching and learning. Digital Education Review, (30):17-29.

Sandu, N. and Gide, E. (2019). Adoption of ai-chatbots to enhance student learning experience in higher education in india. In 2019 18th International Conference on Information Technology Based Higher Education and Training (ITHET), pages 1-5. IEEE.

Smutny, P. and Schreiberova, P. (2020). Chatbots for learning: A review of educational chatbots for the facebook messenger. Computers \& Education, 151:103862.

Vladoiu, M. and Constantinescu, Z. (2020). Learning during covid-19 pandemic: Online education community, based on discord. In 2020 19th RoEduNet Conference: Networking in Education and Research (RoEduNet), pages 1-6. IEEE.

Zhang, L. and Nouri, J. (2019). A systematic review of learning computational thinking through scratch in k-9. Computers \& Education, 141:103607. 